OPEN ACCESS

Edited by:

Ashok Kumar,

University of Florida, United States

Reviewed by:

Muzamil Ahmad,

Indian Institute of Integrative Medicine

(CSIR), India

Adel Helmy

University of Cambridge,

United Kingdom

*Correspondence:

Xin Zhang

zhangxsp@163.com

Chunhua Hang

hang_neurosurgery@163.com

${ }^{\dagger}$ These authors have contributed equally to this work.

Specialty section: This article was submitted to

Neuropharmacology,

a section of the journal

Frontiers in Neuroscience

Received: 04 July 2017 Accepted: 18 October 2017 Published: 03 November 2017

Citation:

Zhang X, Wu Q, Zhang Q, LU Y, LiU J, Li W, Lv S, Zhou M, Zhang $X$ and Hang C (2017) Resveratrol Attenuates Early Brain Injury after Experimental Subarachnoid Hemorrhage via Inhibition of NLRP3 Inflammasome Activation. Front. Neurosci. 11:611. doi: 10.3389/fnins.2017.00611

\section{Resveratrol Attenuates Early Brain Injury after Experimental Subarachnoid Hemorrhage via Inhibition of NLRP3 Inflammasome Activation}

\author{
Xiangsheng Zhang ${ }^{1 \dagger}$, Qi Wu ${ }^{1 \dagger}$, Qingrong Zhang ${ }^{1 \dagger}$, Yue $\mathrm{Lu}^{1}$, Jingpeng $\mathrm{Liu}^{2}$, Wei $\mathrm{Li}^{1}$, \\ Shengyin Lv ${ }^{2}$, Mengliang Zhou ${ }^{1}$, Xin Zhang ${ }^{1,2 *}$ and Chunhua Hang ${ }^{1,2 *}$

\footnotetext{
${ }^{1}$ Department of Neurosurgery, Jinling Hospital, School of Medicine, Nanjing University, Nanjing, China, ${ }^{2}$ Department of Neurosurgery, Jinling Hospital, School of Medicine, Southern Medical University, Nanjing, China
}

Previous studies have demonstrated resveratrol (RSV) has beneficial effects in early brain injury $(\mathrm{EBI})$ after subarachnoid hemorrhage $(\mathrm{SAH})$. However, the beneficial effects of RSV and the underlying mechanisms have not been clearly identified. The nucleotide-binding oligomerization domain-like receptor family pyrin domain-containing 3 (NLRP3) inflammasome activation plays a crucial role in the EBI pathogenesis. The aim of this study was to investigate the role of RSV on the NLRP3 inflammasome signaling pathway and $\mathrm{EBI}$ in rats after $\mathrm{SAH}$. A prechiasmatic cistern injection model was established in rats, and the primary cultured cortical neurons were stimulated with oxyhemoglobin (oxyHb) to induce SAH in vitro. It showed that the NLRP3 inflammasome components, including NLRP3, apoptosis-associated speck-like protein containing

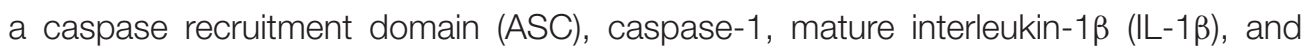
interleukin-18 (IL-18) were upregulated after SAH, and the enhanced NLRP3 after SAH was mainly located in microglia. Treatment with 60 or $90 \mathrm{mg} / \mathrm{kg}$ RSV after SAH dramatically inhibited the expression of NLRP3, but there was no significant difference in the expression of NLRP3 between the SAH $+60 \mathrm{mg} / \mathrm{kg}$ RSV and SAH +90 $\mathrm{mg} / \mathrm{kg}$ RSV groups. In addition, treatment with $30 \mathrm{mg} / \mathrm{kg}$ RSV did not significantly reduced the expression of NLRP3. We next evaluated the neuroprotective effects of RSV against SAH. We determined that SAH-induced NLRP3 inflammasome activation was significantly inhibited in the SAH $+60 \mathrm{mg} / \mathrm{kg}$ RSV group. Meanwhile, $60 \mathrm{mg} / \mathrm{kg}$ RSV administration could markedly inhibit microglia activation and neutrophils infiltration after SAH. Concomitant with the decreased cerebral inflammation, RSV evidently reduced cortical apoptosis, brain edema, and neurobehavioral impairment after SAH. In vitro experiments, RSV treatment also clearly protected primary cortical neurons against oxyHb insults, including reduced the proportion of neuronal apoptosis, alleviated 
neuronal degeneration, and improved cell viabilities. These in vitro data further confirm that RSV has an efficient neuroprotection against SAH. Taken together, these in vivo and in vitro findings suggested RSV could protect against $\mathrm{EBI}$ after $\mathrm{SAH}$, at least partially via inhibiting NLRP3 inflammasome signaling pathway.

Keywords: subarachnoid hemorrhage, early brain injury, resveratrol, inflammation, NLRP3

\section{INTRODUCTION}

Subarachnoid hemorrhage (SAH) is a life-threatening disease and accounts for about $6-8 \%$ of all human strokes (Sehba et al., 2011; Li J. et al., 2015). Currently, early brain injury (EBI) is one of the key mechanisms of SAH and plays a critical role in high mortality and disability after SAH (Sehba et al., 2011; Chen et al., 2014; Yuan et al., 2017). A growing body of evidence indicates that inflammatory injury plays pivotal roles in the EBI pathogenesis and may represent for a promising goal for therapeutic intervention following SAH (You et al., 2016; Zhang et al., 2016b; Wu Q. et al., 2017).

Resveratrol (RSV), a natural occurring polyphenolic compound extracted from pines and grapevines, can easily pass the blood-brain barrier (Zhang et al., 2016a). Its wide range of biological properties, such as anti-inflammatory, anti-oxidant, and anti-tumor activity, has been extensively studied in different research fields (Fu et al., 2013; Shao et al., 2014; Zhang T. et al., 2014). In the central nervous system (CNS) diseases including traumatic brain injury (TBI), cerebral ischemia and neurodegenerative diseases, RSV has been shown to have promising neuroprotective effects due to its multiple functions (Della-Morte et al., 2009; Lin et al., 2014; Rege et al., 2014). Furthermore, recent researches have also demonstrated that RSV protects against EBI after SAH due to its powerful anti-inflammatory property (Shao et al., 2014; Zhang et al., 2016a). However, the anti-inflammatory effects of RSV and the potential molecular mechanisms have not been fully investigated in EBI after SAH.

The nucleotide-binding oligomerization domain-like receptor (NLR) family pyrin domain-containing 3 (NLRP3) inflammasome, a core component of the innate immune system, can facilitate caspase-1, interleukin-1 $\beta$ (IL-1 $\beta$ ) and interleukin-18 (IL-18) processing, and then amplify the inflammatory response (Chen et al., 2013). Recently, mounting evidence has demonstrated NLRP3 inflammasome plays a key role in a variety of inflammatory diseases, such as atherosclerosis, metabolic syndrome, cardiovascular disorders, and stroke (Chen et al., 2013; Coll et al., 2015). In SAH, NLRP3 inflammasome activation is also essential for the modulation of pro-inflammatory cytokines, and inhibition NLRP3 inflammasome by pharmacological treatment could protect against brain injury after SAH (Chen et al., 2013; Li J. et al., 2015; Dong et al., 2016). In addition, the activation of NLRP3 inflammasome is associated with the frequency of apoptosis, and inhibition NLRP3 inflammasome could suppress cell apoptosis (Wali et al., 2013; Wu et al., 2014; Lebeaupin et al., 2015; Li Y. et al., 2015; Dong et al., 2016). However, until now, no study has been performed to investigate whether RSV could influence NLRP3 inflammasome-related inflammatory pathway during SAH. Thus, the aim of the present study was to explore the role of NLRP3 inflammasome in the therapeutic effects of RSV in EBI after SAH.

\section{MATERIALS AND METHODS}

\section{Animals}

Adult male Sprague-Dawley rats weighing $250-300 \mathrm{~g}$ and 15-18-day-old pregnant C57BL/6 mice were purchased from the Animal Center of Jinling Hospital (Nanjing, China). All experimental protocols were approved by the Animal Care and Use Committee of Nanjing University and in accordance with the Guide for the Care and Use of Laboratory Animals by National Institutes of Health (Wu L. Y. et al., 2017).

\section{Primary Neuron Culture}

Primary cortical neurons were cultured as described previously (Sun et al., 2014; Wang Z. et al., 2016). Briefly, cerebral cortex was isolated from brains of fetal mice. The blood vessels and meninges were removed and brain tissues were digested with $0.25 \%$ trypsin for $5 \mathrm{~min}$ at $37^{\circ} \mathrm{C}$. Then, the supernatant containing trypsin was discarded and washed with pre-cooling phosphate buffered saline (PBS). After that, brain tissue suspensions were passed through a $22 \mu \mathrm{m}$-filter and centrifuged at 1,500 r/min for $5 \mathrm{~min}$. Subsequently, the cells were distributed in poly-D-lysine-coated plates and suspended in Neurobasal media supplemented with B27, glutamate, Hepes, penicillin and streptomycin (all bought from Gibco Incorporation). Cultures were maintained in a humidified incubator with $5 \%$ $\mathrm{CO}_{2}$ and $95 \%$ air at $37^{\circ} \mathrm{C}$. Finally, half of the medium was replaced with fresh medium every 2 days. The in vitro studies were performed with neurons that had been in culture for 8-10 days.

\section{SAH Rat Model}

In vivo experiments, the prechiasmatic cistern injection model was prepared according to previous studies (Figure 1F) (Li J. et al., 2015; Yan et al., 2015; Zhang X. S. et al., 2015). Briefly, after the rats were anesthetized with $10 \%$ chloral hydrate $(0.35$ $\mathrm{ml} / 100 \mathrm{~g}$ ), a total of $0.35 \mathrm{ml}$ of non-heparinized fresh autologous arterial blood from the femoral artery was slowly (in the course of $20 \mathrm{~s}$ ) injected into the prechiasmatic cistern under aseptic conditions (Zhang X. S. et al., 2015). After the procedures, the animals were kept in a $30^{\circ}$, heads-down position for $20 \mathrm{~min}$. Rats were returned to their feeding room after wake up from anesthesia.

In vitro experiments, primary cultured cortical neurons were stimulated with oxyhemoglobin (oxyHb, Ruibio, O7109). Primary cultured neurons were prepared as mentioned above. oxyHb was resolved into $10 \mu \mathrm{M}$ with culture medium, which was 
determined according to previous study (Wang Z. et al., 2016). Neurons were stimulated with $10 \mu \mathrm{M}$ oxyHb for $24 \mathrm{~h}$ to mimic SAH condition.

\section{Study Design}

\section{Experiment 1}

For in vivo experiments, rats were randomly assigned to the various groups: sham group $(n=36)$; sham $+60 \mathrm{mg} / \mathrm{kg} \mathrm{RSV}$ group $(n=36)$; SAH group $(n=12)$; $\mathrm{SAH}+$ vehicle group $(n=36) ; \mathrm{SAH}+30 \mathrm{mg} / \mathrm{kg} \mathrm{RSV}$ group $(n=12), \mathrm{SAH}+60 \mathrm{mg} / \mathrm{kg}$ RSV group $(n=36)$, and SAH $+90 \mathrm{mg} / \mathrm{kg} \mathrm{RSV} \mathrm{group}(n=12)$. All rats were sacrificed to collect brain samples at $24 \mathrm{~h}$ after SAH. Post-treatment assessments included neurological scores, brain edema, Western blot analysis, double immunofluorescence staining, and TUNEL staining.

\section{Experiment 2}

For in vitro experiments, primary cultured cortical neurons were randomly divided into six groups: control group, control + $20 \mu \mathrm{M}$ RSV group, SAH + vehicle group, SAH + $1 \mu \mathrm{M}$ RSV group, $\mathrm{SAH}+10 \mu \mathrm{M}$ RSV group, and SAH $+20 \mu \mathrm{M}$ RSV group. The neurons were collected for Western blot analysis, cell viability analysis, and histopathological study.

\section{Drug Administration}

For in vivo experiments, RSV (Sigma-Aldrich, St. Louis, MO, USA) was dissolved in $1 \%$ dimethylsulfoxide (DMSO) and physiological saline. In the rats of sham + RSV or SAH + RSV groups, RSV was injected intraperitoneally at different doses (30, $60,90 \mathrm{mg} / \mathrm{kg}$ ) at 2 and $12 \mathrm{~h}$ post-injury (Zhang et al., 2016a). The doses were determined based on previous studies (Shao et al., 2014; Zhang et al., 2016a). Rats in SAH + vehicle group received an intraperitoneal injection of the same volume of vehicle at the corresponding time point. For in vitro experiments, RSV was dissolved in DMSO and then added to neuronal medium to reach different final concentrations $(1 \mu \mathrm{M}, 10 \mu \mathrm{M}$, and $20 \mu \mathrm{M})$. The concentrations of RSV used in in vitro study were based on previous studies in vitro (Wang et al., 2015; Zhang Q. et al., 2015).

\section{Western Blot}

Western blot was carried out as previously described (Zhang X. S. et al., 2015). Briefly, protein samples were separated by $10 \%$ SDS-PAGE and electrophoretically transferred to polyvinylidenedifluoride (PVDF) membrane (Bio-Rad Lab, Hercules, CA, USA). After the PVDF membrane was blocked for $2 \mathrm{~h}$, the membrane was then incubated with primary antibodies overnight (Zhang X. S. et al., 2015). The primary antibodies against NLRP3 (cat\# SC-66846), ASC (cat\# SC-22514), caspase1 (cat\# SC-398715), IL-1 $\beta$ (cat\# SC-32294), IL-18 (cat\# SC7954), TNF- $\alpha$ (cat\# SC-52746), Bcl-2 (cat\# SC-492), and Bax (cat\# SC-526) were bought from Santa Cruz Biotechnology. Antibody against caspase-3 (cat\# 9661) was purchased from Cell Signaling Technology. After that, the membranes were incubated in the appropriate horseradish peroxidase (HRP)-conjugated secondary antibody. The blotted protein bands were developed using enhanced chemiluminescence kit (Amersham, Arlington Heights, IL, USA).

\section{Immunohistochemistry}

Briefly, brain sections ( $4 \mu \mathrm{m}$ thickness) were incubated overnight at $4{ }^{\circ} \mathrm{C}$ with primary antibody against NLRP3, ASC, and caspase1. After washing carefully in PBS for about $15 \mathrm{~min}$, the sections were then incubated with HRP-conjugated secondary antibody for $1 \mathrm{~h}$ at room temperature. 3,3'-diaminobenzidine (DAB) was used to visualize NLRP3, ASC, and caspase-1. The intensity of staining in each section was evaluated by using a five-grades scoring system (Wang et al., 2012; Zhang X. S. et al., 2015). " 0 " indicates that there were no detectable positive cells; " 1 " represents very low density of positive cells; " 2 " represents a moderate density of positive cells; " 3 " represents the higher, but not maximal density of positive cells; and " 4 " represents the highest density of positive cells (Wang et al., 2012; Zhang et al., 2016a).

\section{Immunofluorescence Staining}

Immunofluorescence staining was carried out as described previously (Sun et al., 2014). Frozen tissue sections $(6 \mu \mathrm{m}$ thickness) were incubated in blocking buffer for $2 \mathrm{~h}$ at room temperature. After they were washed three times with PBS, sections were incubated with primary antibodies and corresponding secondary antibodies. After washing with PBS for three times, the slides were stained with 4-diamidino-2phenylindole (DAPI) for $2 \mathrm{~min}$. Images were obtained using a ZEISS HB050 inverted microscope system and the fluorescently stained cells were analyzed using Image J program.

\section{TUNEL Staining}

Terminal deoxynucleotidyl transferase-mediated dUTP nick-end labeling (TUNEL) staining was performed according to the manufacturer's instruction (Roche, South San Francisco, CA, USA). In brief, after brain sections or cultured neurons were incubated with primary antibody at $4^{\circ} \mathrm{C}$ overnight, the slides or cultured neurons were incubated with TUNEL reaction mixture for $1 \mathrm{~h}$ at $37^{\circ} \mathrm{C}$. The slides were washed several times with PBS and then stained with DAPI for $2 \mathrm{~min}$. The quantification of TUNEL-positive neurons was performed by a pathologist blinded to the experiments groups.

\section{Neurological Scores and Brain Water Content}

The neurological scores were recorded $24 \mathrm{~h}$ after SAH with a 6-point scoring system (Table 1; Zhang et al., 2016a). Brain water content was evaluated as previously reported (Zhang et al., 2016a). Briefly, brains were removed and separated into cerebrum, cerebellum and brain stem. Each part was weighed immediately to obtain the wet weight and dried for $72 \mathrm{~h}$ at $100^{\circ} \mathrm{C}$ to obtain the dry weight. The percentage of water content was calculated as follows: [(wet weight-dry weight)/wet weight] $\times$ 100\% (Zhang et al., 2016a).

\section{Cell Viability Analysis}

The primary cultured neurons viability was evaluated by measuring the lactate dehydrogenase (LDH) activity. LDH activity was determined with an assay kit according to the 
TABLE 1 | Behavior scores.

\begin{tabular}{llc}
\hline Category & Behavior & Score \\
\hline Appetite & Finished meal & 0 \\
& Left meal unfinished & 1 \\
& Scarcely ate & 2 \\
Activity & Walk and reach at least three corners of the cage & 0 \\
& Walk with some stimulation & 1 \\
& Almost always lying down & 2 \\
Deficits & No deficits & 0 \\
& Unstable walk & 1 \\
& Impossible to walk & 2 \\
\hline
\end{tabular}

manufacturers' protocol (Beyotime Biotechnology, Shanghai, China).

\section{Statistical Analysis}

Data were presented as mean \pm S.E.M. SPSS 19.0 was used for statistical analysis of the data. Statistical analysis between two groups were performed with the Student's $t$-test and between multiple groups with one-way analysis of variance (ANOVA) followed by Tukey post-hoc test. Statistical significance was inferred at $P<0.05$.

\section{RESULTS}

\section{General Observations and Mortality Rate}

There were no statistical differences in mean arterial blood pressure and heart rate among all experimental groups. No animals died in the sham group (0/36 rats) and sham $+60 \mathrm{mg} / \mathrm{kg}$ RSV group (0/36 rats). The mortality rate of the rats was $25.0 \%$ (3/12 rats) in the SAH group; $19.4 \%$ (7/36 rats) in the SAH + vehicle group; $25 \%$ (3/12 rats) in the $\mathrm{SAH}+30 \mathrm{mg} / \mathrm{kg} \mathrm{RSV}$ group; $13.9 \%$ (5/36 rats) in the SAH $+60 \mathrm{mg} / \mathrm{kg}$ RSV group; $16.7 \%(2 / 12$ rats) in the $\mathrm{SAH}+90 \mathrm{mg} / \mathrm{kg}$ RSV group.

\section{NLRP3 Inflammasome Components Were Upregulated at $24 \mathrm{~h}$ Post-SAH}

Western blot results showed that NLRP3 components, including NLRP3 (0.66 \pm 0.04 vs. $0.98 \pm 0.06)$, ASC ( $0.59 \pm 0.06$ vs. 0.91 $\pm 0.06)$, caspase- 1 ( $0.69 \pm 0.05$ vs. $0.85 \pm 0.05)$, IL- $1 \beta(0.43 \pm$ 0.04 vs. $0.58 \pm 0.04)$, and IL- $18(0.57 \pm 0.03$ vs. $0.69 \pm 0.04)$ were evidently increased at $24 \mathrm{~h}$ post-SAH (Figures 1A,B). The distribution of NLRP3 in the brain cortex was further identified by immunohistochemistry and immunofluorescence staining (Figures 1C-E). As shown, NLRP3 was weakly expressed in the sham group. In contrast, NLRP3 was evidently increased in the cortex at $24 \mathrm{~h}$ post-SAH $(1.21 \pm 0.18$ vs. $2.08 \pm 0.28)$, and the increased NLRP3 was mainly located in microglia after SAH.

\section{Effects of RSV on the NLRP3 Inflammasome Signaling Pathway}

As shown in Figure 2A, both $60 \mathrm{mg} / \mathrm{kg}$ and $90 \mathrm{mg} / \mathrm{kg} \mathrm{RSV}$ treatment significantly reduced the enhanced expression of NLRP3 in brain cortex after SAH $(0.99 \pm 0.07$ vs. $0.77 \pm 0.04$,
$0.99 \pm 0.07$ vs. $0.76 \pm 0.05)$. However, $30 \mathrm{mg} / \mathrm{kg}$ RSV treatment did not inhibit the expression of NLRP3 when compared with that in the SAH + vehicle group $(0.99 \pm 0.07$ vs. $0.95 \pm 0.06)$. In addition, we noted that there was no statistical difference in the expression of NLRP3 between the SAH $+60 \mathrm{mg} / \mathrm{kg}$ RSV and $\mathrm{SAH}+90 \mathrm{mg} / \mathrm{kg}$ RSV groups $(0.77 \pm 0.04$ vs. $0.76 \pm 0.05)$. Immunofluorescence staining results (Figures 2B,C) was similar to that of Western blot, suggesting that $60 \mathrm{mg} / \mathrm{kg}$ RSV treatment could efficiently inhibit NLRP3 expression after SAH. Therefore, we chose $60 \mathrm{mg} / \mathrm{kg}$ RSV in the following experiments.

We next evaluated the effects of RSV on the NLRP3 inflammasome signaling pathway. Western blot results (Figures 3A-G) showed that the protein expressions of NLRP3 ( $0.66 \pm 0.04$ vs. $0.99 \pm 0.07)$, ASC $(0.59 \pm 0.06$ vs. $0.93 \pm$ $0.07)$, caspase- $1(0.67 \pm 0.04$ vs. $0.85 \pm 0.05), \mathrm{IL}-1 \beta(0.42 \pm 0.04$ vs. $0.60 \pm 0.04)$, IL- $18(0.56 \pm 0.03$ vs. $0.69 \pm 0.04)$, and TNF- $\alpha$ $(0.57 \pm 0.07$ vs. $1.04 \pm 0.10)$ were markedly upregulated at $24 \mathrm{~h}$ after SAH. In contrast, administration of RSV significantly reduced the expression of NLRP3 inflammasome components as compared with the SAH + vehicle group $(0.99 \pm 0.07$ vs. $0.77 \pm 0.04,0.93 \pm 0.07$ vs. $0.64 \pm 0.06,0.85 \pm 0.05$ vs. $0.67 \pm$ $0.04,0.60 \pm 0.04$ vs. $0.42 \pm 0.04,0.69 \pm 0.04$ vs. $0.56 \pm 0.03$, $1.04 \pm 0.10$ vs. $0.72 \pm 0.07)$. Similarly, immunohistochemistry (Figures 4A,B) also revealed elevated NLRP3, ASC, and caspase1 immunoreactivities in the brain cortex after $\mathrm{SAH}$, which were also reversed by RSV treatment $(2.13 \pm 0.23$ vs. $1.38 \pm 0.21$, $1.96 \pm 0.24$ vs. $1.21 \pm 0.16,1.92 \pm 0.19$ vs. $1.29 \pm 0.15)$. These results suggested that RSV could inhibit NLRP3 inflammasome activation after $\mathrm{SAH}$.

\section{Effects of RSV on Microglia Activation and Neutrophils Infiltration at $24 \mathrm{~h}$ Post-SAH}

Activation of microglia and neutrophils infiltration play important roles in inflammatory response after SAH. Our results showed that the expressions of Iba-1 (0.35 \pm 0.04 vs. $0.69 \pm$ $0.06)$ and MPO $(0.48 \pm 0.06$ vs. $0.79 \pm 0.05)$ were higher at $24 \mathrm{~h}$ after $\mathrm{SAH}$ insults than that in the sham group (Figures $\mathbf{5 A - C}$ ). In addition, the number of Iba-1- and MPO-positive cells in the brain cortex was also significantly enhanced after SAH $(7.75 \pm 1.04$ vs. $16.13 \pm 1.21,4.25 \pm 0.66$ vs. $9.83 \pm 0.84)$ (Figures 5D-G). After administration with RSV, the upregulated Iba- $1(0.69 \pm 0.06$ vs. $0.51 \pm 0.04)$ and MPO $(0.79 \pm 0.05$ vs. $0.54 \pm 0.05)$ expression as well as the Iba-1- (16.13 \pm 1.21 vs. $11.13 \pm 1.24)$ and MPO-positive $(9.83 \pm 0.84$ vs. $6.17 \pm 1.05)$ cells were markedly reduced when compared with the SAH + vehicle group (Figures 5A-G). These results suggested that RSV could inhibit inflammatory response in the brain after SAH.

\section{Influence of RSV on Neuronal Apoptosis, Brain Water Content, and Neurobehavioral Impairment at $\mathbf{2 4} \mathrm{h}$ after SAH}

Western blot results showed that RSV treatment significantly reduced $\operatorname{Bax}(0.75 \pm 0.05$ vs. $0.47 \pm 0.05)$ and caspase-3 (0.57 \pm 0.05 vs. $0.34 \pm 0.05)$ protein levels, and enhanced the diminished level of $\mathrm{Bcl} 2(0.85 \pm 0.06$ vs. $1.11 \pm 0.06)$ compared with the $\mathrm{SAH}+$ vehicle group (Figures 6A,B). TUNEL staining further 


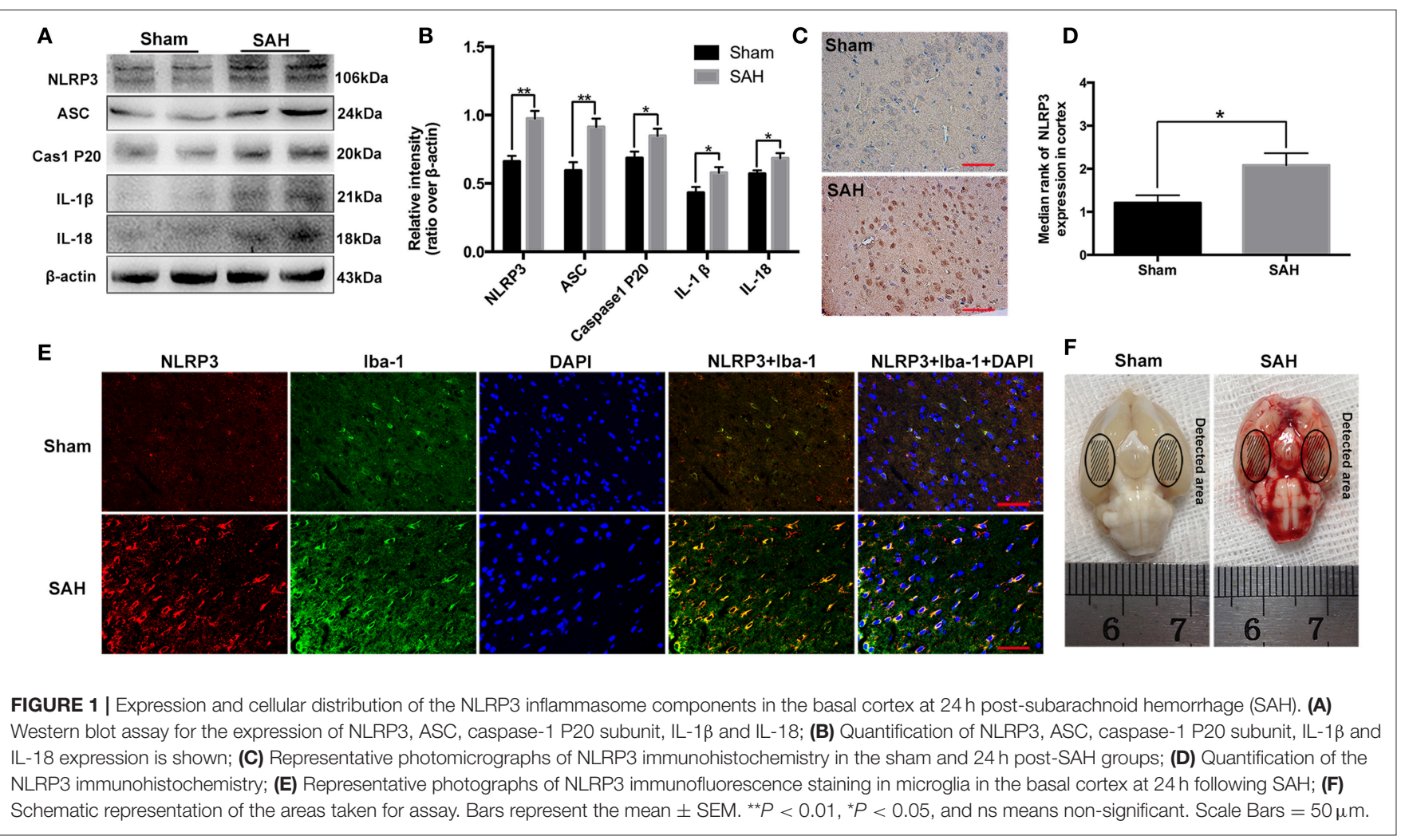

showed that RSV administration could increase the proportion of surviving neurons after SAH $(25.15 \pm 3.07$ vs. $16.67 \pm$ 1.48) (Figures 6C,D). Brain water content and neurobehavioral function were blindly investigated at $24 \mathrm{~h}$ following SAH. As shown (Figures 6E,F), brain edema and neurological impairment were significantly aggravated after SAH insults compared with the sham group $(0.788 \pm 0.098$ vs. $0.798 \pm 0.240,0.61 \pm 0.14$ vs. $2.78 \pm 0.33)$. In contrast, RSV treatment could partly reverse the aggravated brain edema and neurological impairment after SAH $(0.798 \pm 0.240$ vs. $0.792 \pm 0.127,2.78 \pm 0.33$ vs. $1.83 \pm 0.23)$.

\section{Effects of RSV on Neuronal Damage in Primary Cortical Neurons in Vitro}

As shown in Figure 7A, neurons exposed to oxyHb showed evident neuronal degeneration characterized by swollen neuronal cell bodies with loss of synapses and brightness. Compared with $\mathrm{SAH}+$ vehicle group, RSV treatment significantly prevented these morphological changes in a dose-dependent manner. We then evaluated the influence of different doses of RSV on apoptotic pathways, TUNEL-apoptosis, and $\mathrm{LDH}$ release in primary cortical neurons (Figures 7B-F). Results showed that 10 or $20 \mu \mathrm{M}$ RSV treatment evidently inhibited apoptotic pathways, reduced the number of TUNEL-positive neurons $(75.51 \pm 4.60$ vs. $53.97 \pm 4.31,75.51 \pm 4.60$ vs. $45.60 \pm 3.44)$, and decreased LDH activity $(166.75 \pm 3.87$ vs. $145.12 \pm 4.57,166.75 \pm$ 3.87 vs. $135.67 \pm 3.60)$ after oxyHb stimulation in a dosedependent manner. However, $1 \mu \mathrm{M}$ RSV treatment did not alleviate neuronal damage when compared with SAH + vehicle group. One possible reason could be that $1 \mu \mathrm{M}$ RSV might not be sufficient to produce protection against neuronal damage exposed to oxyHb. These results suggested that RSV treatment could also reduce neuronal damage in SAH model in vitro.

\section{DISCUSSION}

In the current study, we evaluated the NLRP3 inflammasome activation responded to brain injury following $\mathrm{SAH}$, and its relationship with the RSV's neuroprotective effects in experimental SAH models. The major findings were as follows: (1) NLRP3 inflammasome was upregulated in the brain cortex after SAH, and the enhanced NLRP3 after SAH was mainly expressed in microglia; (2) RSV treatment inhibited NLRP3 inflammasome activation after $\mathrm{SAH}$, and suppressed the microglia activation and neutrophils infiltration; (3) administration with RSV reduced neuronal cell apoptosis, brain edema, and neurological impairment after SAH; (4) RSV significantly alleviated neuronal damage, prevented neurodegeneration, and improved cell viabilities in primary cortical neurons with a dose-dependent manner.

Inflammation has been proved to play key roles in the pathophysiology of SAH-induced brain injury (Zhang et al., 2016a,b; Wu Q. et al., 2017). At the onset of SAH, the infiltration of blood-borne leukocyts is one of the earliest events (Ma et al., 2014). Microglia, the primary resident macrophages of the CNS, can be activated by the infiltrated leukocytes after SAH. Subsequently, the infiltrated leukocytes and activated microglia 

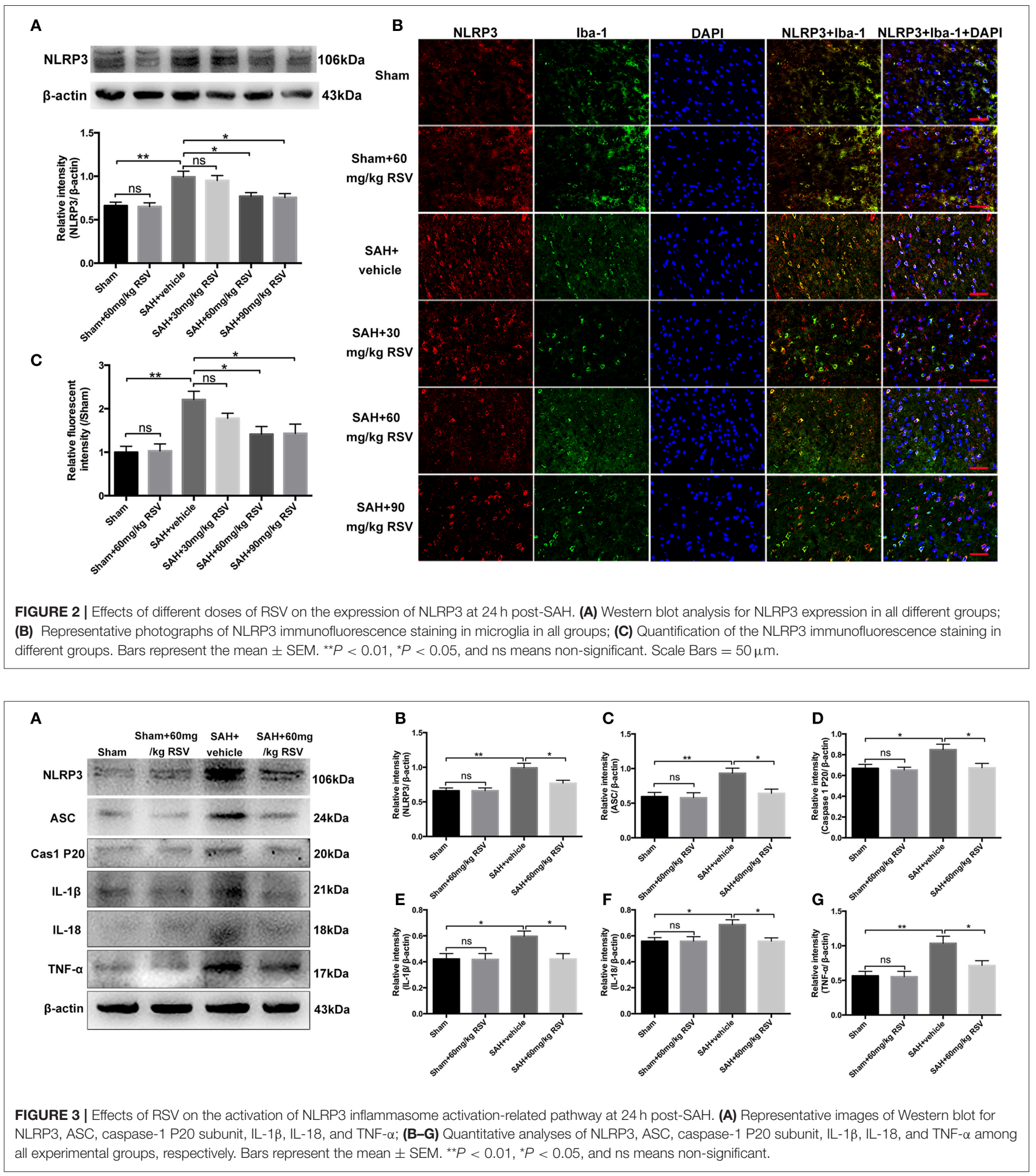

produce and release a number of pro-inflammatory cytokines to cause direct damage to surrounding neural cells and amplify leukocyte recruitment to further aggravate brain damage after SAH (Chen et al., 2013). However, the mechanisms by which
SAH stimulates the inflammatory response have not been fully elucidated.

Recently, there is a wealth of studies have shown that inflammasome is involved in the pathological process of various 


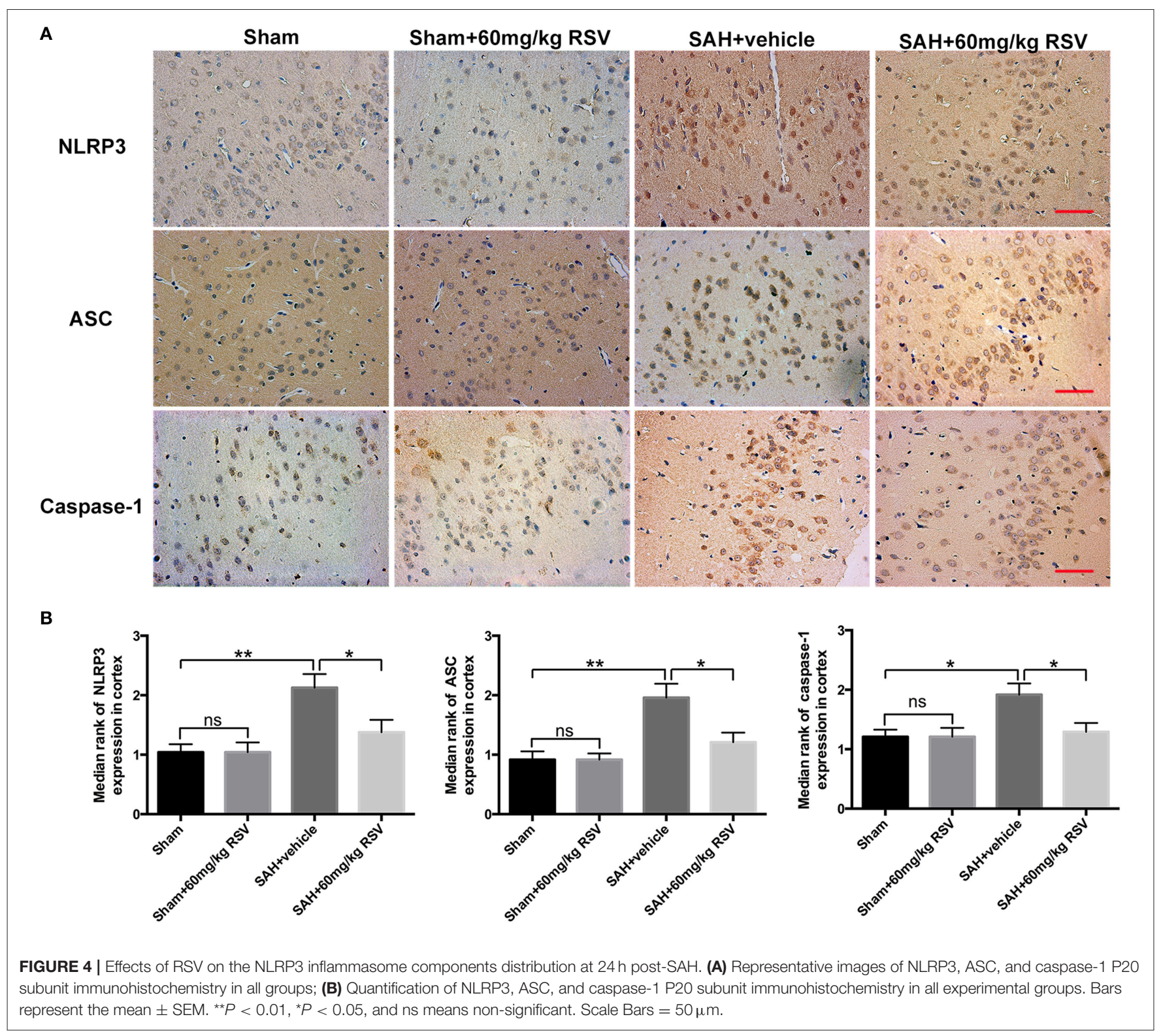

sterile inflammatory disorders, such as gout, Crohn disease, systemic-onset juvenile arthritis, and cryopyrin-associated periodic syndromes (Walsh et al., 2014; Coll et al., 2015; Zhu et al., 2017). The inflammasome is a large multiple protein complex that modulates maturation and release of the pro-inflammatory cytokines (Chen et al., 2013). Among all inflammasomes, the NLRP3 inflammasome is the best studied from this family (Ma et al., 2014; Guo et al., 2016). The NLRP3 inflammasome contains NLR protein cryopyrin, the adapter ASC (apoptosis-associated speck-like protein containing a caspase recruitment domain), and pro-caspase-1(Wang Y. et al., 2016). Upon activation, aberrant NLRP3 inflammasome results in a robust release of mature IL-1 $\beta$ and IL-18, which plays key roles in a variety of diseases, including CNS disorders (Chen et al., 2013; Ma et al., 2014; Coll et al., 2015).
In experimental ischemic stroke models, Yang et al reported that NLRP3 deficiency alleviated neurovascular damage via attenuation of inflammatory pathways (Yang et al., 2014). Ma et al also reported that NLRP3 inflammasome amplified the inflammatory response following experimental intracerebral hemorrhage (ICH) model, and NLRP3 knockdown reduced inflammatory damage and improved neurological functions following ICH (Ma et al., 2014). Notably, recent studies have observed that NLRPP3 inflammasome contributes to inflammatory damage following $\mathrm{SAH}$, and inhibition NLRP3 inflammasome by hydrogen-rich saline, melatonin and minocycline could ameliorate EBI after SAH (Chen et al., 2013; Li J. et al., 2015; Shao et al., 2015; Dong et al., 2016). In the current study, we also observed that the NLRP3 inflammasome components were upregulated after SAH. In addition, the 


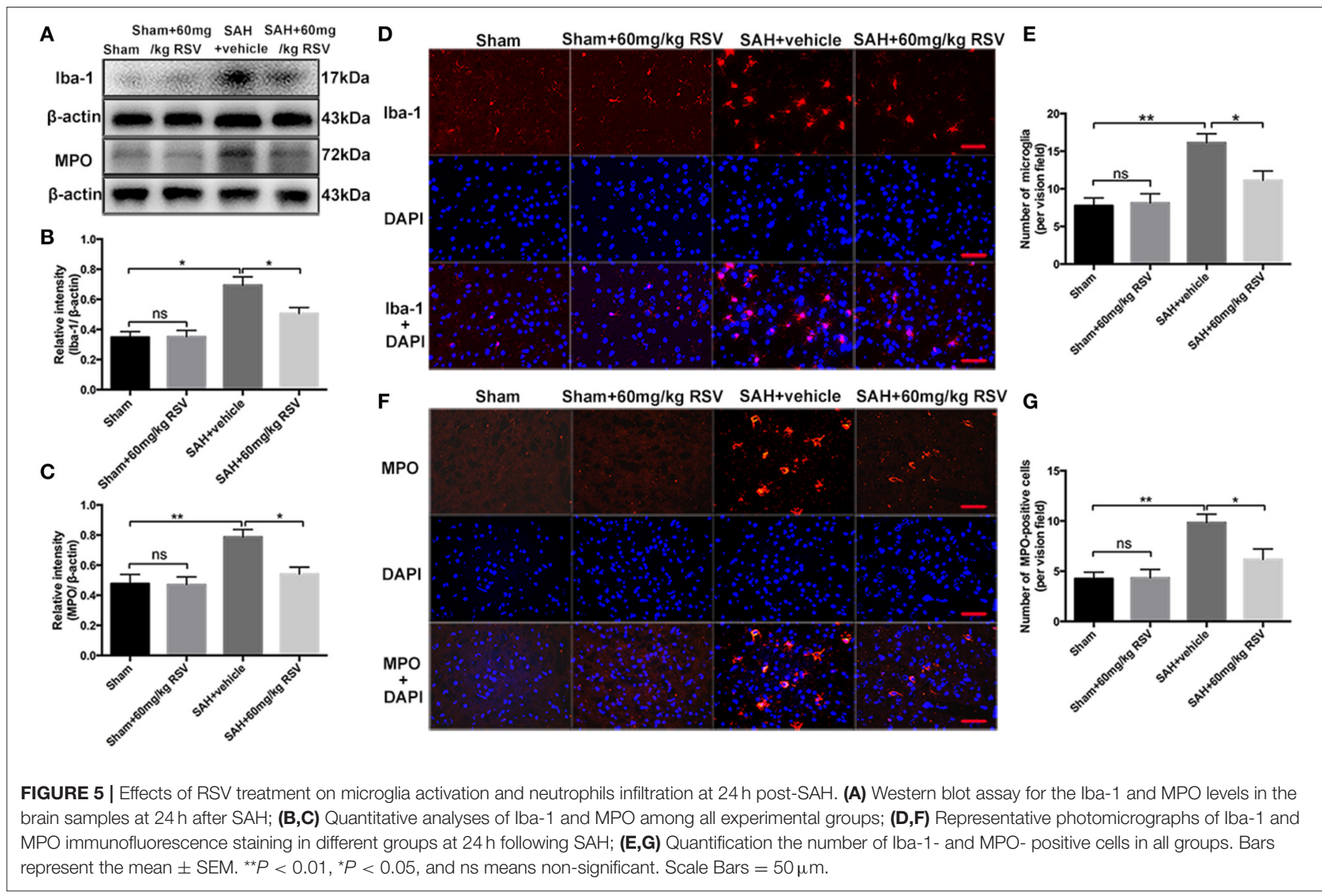

enhanced NLRP3 after SAH was mainly distributed in microglia. These results were consistent with previous studies suggesting that NLRP3 inflammasome may play a pivotal role in the pathologic process of SAH and inhibiting NLRP3 inflammasome activation might be a useful therapeutic intervention following SAH (Chen et al., 2013; Li J. et al., 2015).

RSV is a natural polyphenolic compound distributed at high concentrations in grapes, pines, as well as peanuts (Zhou et al., 2014). Recently, a number of studies have highlighted that RSV may be a promising agent in a number of CNS disorders due to its multiple functions, such as anti-inflammatory, anti-oxidant, and neuroprotective effects (Shao et al., 2014; Arteaga et al., 2015; Lopez et al., 2015; Zhang et al., 2016a). RSV has been demonstrated to ameliorate neurovascular damage in ischemic stroke and TBI (Della-Morte et al., 2009; Lin et al., 2014; Koronowski et al., 2015). Importantly, RSV also has beneficial effects against SAH insults via its powerful anti-inflammatory action (Shao et al., 2014; Zhang et al., 2016a). However, the molecular mechanisms underlying the neuroprotective effects of RSV in SAH remain elusive. Previous studies reported that RSV could modulate NLRP3 inflammasome activation in several different research fields (Fu et al., 2013; Yang and Lim, 2014; Sui et al., 2016). Yang et al proved that RSV ameliorated hepatic metaflammation by inhibiting NLRP3 inflammasome activation (Yang and Lim, 2014). Sui et al found that RSV could protect against sepsis-associated encephalopathy via inhibiting NLRP3/IL-1 $\beta$ axis in microglia (Sui et al., 2016). Thus, we speculated that RSV could protect the brain against SAH insults through the inhibition of NLRP3 inflammasome activation.

Our findings were consistent with previous studies (Fu et al., 2013; Yang and Lim, 2014; Sui et al., 2016). We found that RSV treatment markedly inhibited the NLRP3 inflammasome activation and the subsequent production of pro-inflammatory cytokines after SAH. In addition, microglia activation and neutrophil infiltration, which played important roles in cerebral inflammation after $\mathrm{SAH}$, were also suppressed by RSV administration. Cell apoptosis is a key element of EBI after SAH and is responsible for disastrous outcomes following SAH (Sehba et al., 2012; Zhang X. S. et al., 2015). In addition to the powerful anti-inflammatory function, the anti-apoptotic property is also an important part of RSV's protection (Lopez et al., 2015). In the present study, RSV also showed neuroprotective effects via inhibition of neuronal apoptosis. These might be mediated by RSV's anti-inflammatory and anti-apoptosis functions. To further determine the beneficial effects of RSV on neuronal damage after SAH, we evaluated the influence of RSV on primary cortical neurons exposed to oxyHb. As expected, RSV evidently prevented neuronal degeneration, inhibited neuronal apoptosis, and restored cell viabilities in primary cortical neurons in a 


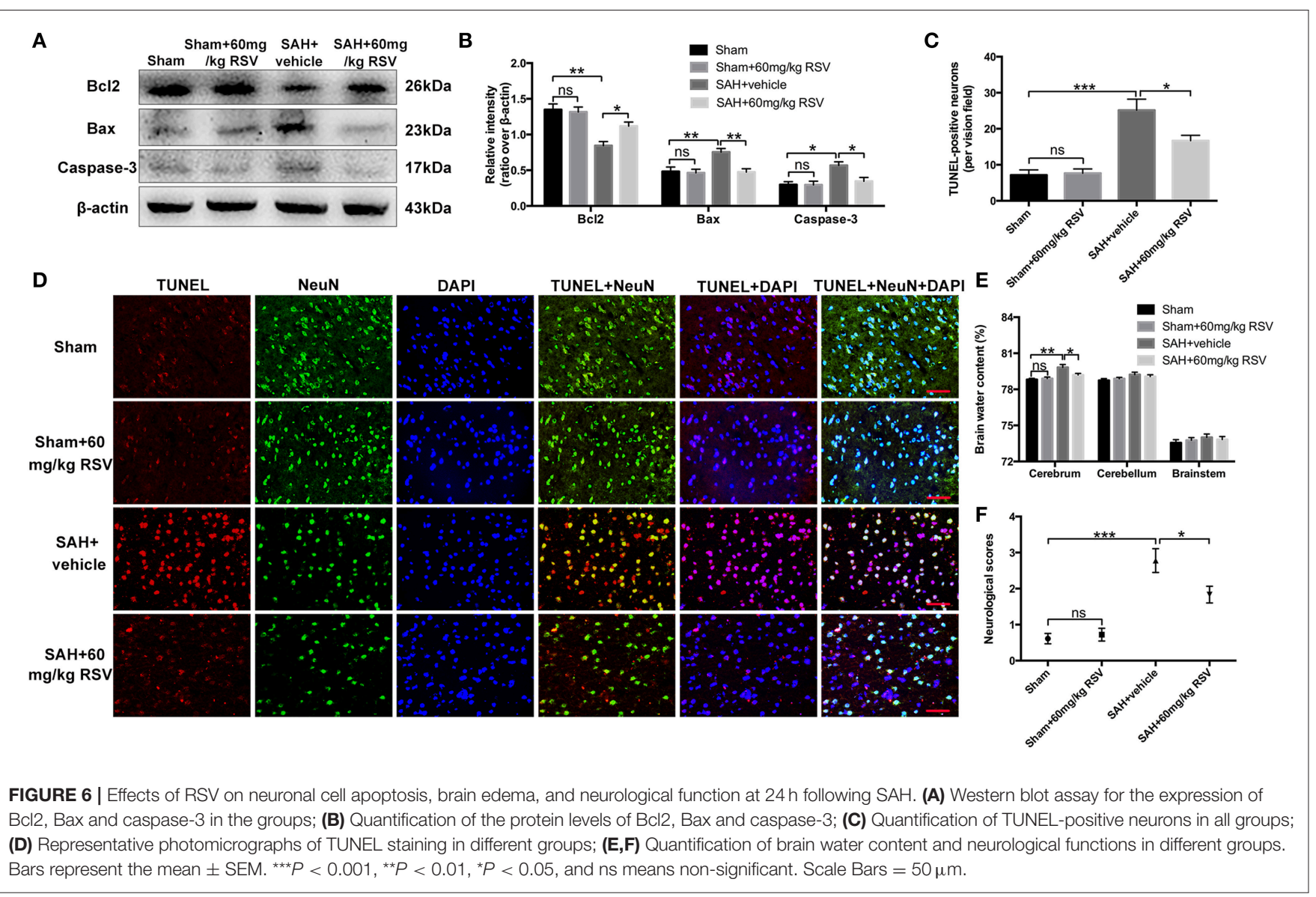

dose-dependent manner, suggesting that RSV was an efficient neuroprotective drug against SAH injury. These findings further support that NLRP3 inflammasome activation plays a key role in the development of EBI after SAH. Meanwhile, the NLRP3 inflammasome inhibition contributed to the profound neuroprotective effects of RSV against EBI induced $\mathrm{SAH}$.

It should be reported that our study has several limitations. Firstly, recent studies reported that microglia have dual phenotypes, pro-inflammatory M1 and anti-inflammatory M2 phenotypes. M1 microglia can increase pro-inflammatory cytokines and upregulate reactive oxygen and nitrogen species. In contrast, M2 microglia release anti-inflammatory cytokines and growth factors, exerting the opposite effects of M1 microglia (Lan et al., 2017b; Yang et al., 2017; Zhang et al., 2017). Studies have shown that activated microglia develop into different phenotypes in different microenvironments and exhibit distinct functions (Lan et al., 2017a; Zhang et al., 2017). In this study, we showed that RSV inhibited microglia activation after SAH. However, we are not sure whether RSV can modulate M2 microglia activation in SAH. Yang et al. reported that RSV could reduce inflammatory damage and promoted microglia polarization to the M2 phenotype in LPS-induced neuroinflammation (Yang et al., 2017). Thus, we speculated that RSV could inhibit microglia activation by promoting microglia polarization toward to the M2 phenotype in SAH. Secondly, the molecular basis underlying NLRP3 inflammasome activation induced by SAH is far from complete. In addition, how RSV regulates NLRP3 inflammasome activation in SAH still remains obscure. Previous studies demonstrated that reactive oxygen species (ROS) was a necessary step for NLRP3 activation, and inhibition ROS production by ROS inhibitor suppressed NLRP3 inflammasome activation (Murphy, 2009). Considering that oxidative damage is a crucial component in EBI pathogenesis, and RSV has a strong anti-oxidant property in other research fields (Zhang X. S. et al., 2014; Zhou et al., 2014; Narayanan et al., 2015; Zhang et al., 2016b), we speculated that RSV might inhibit NLRP3 inflammasome activation by suppressing ROS production. Finally, we could not exclude the possible effects of other pathways participated in the beneficial function of RSV in SAH. Given that the present study is a preliminary research, additional works are still warranted to clarify these issues.

\section{CONCLUSION}

In summary, our study demonstrated that RSV could protect against EBI, at least partly via inhibiting the NLRP3 inflammasome signaling pathway-related inflammatory response. Although more work is required, current data 


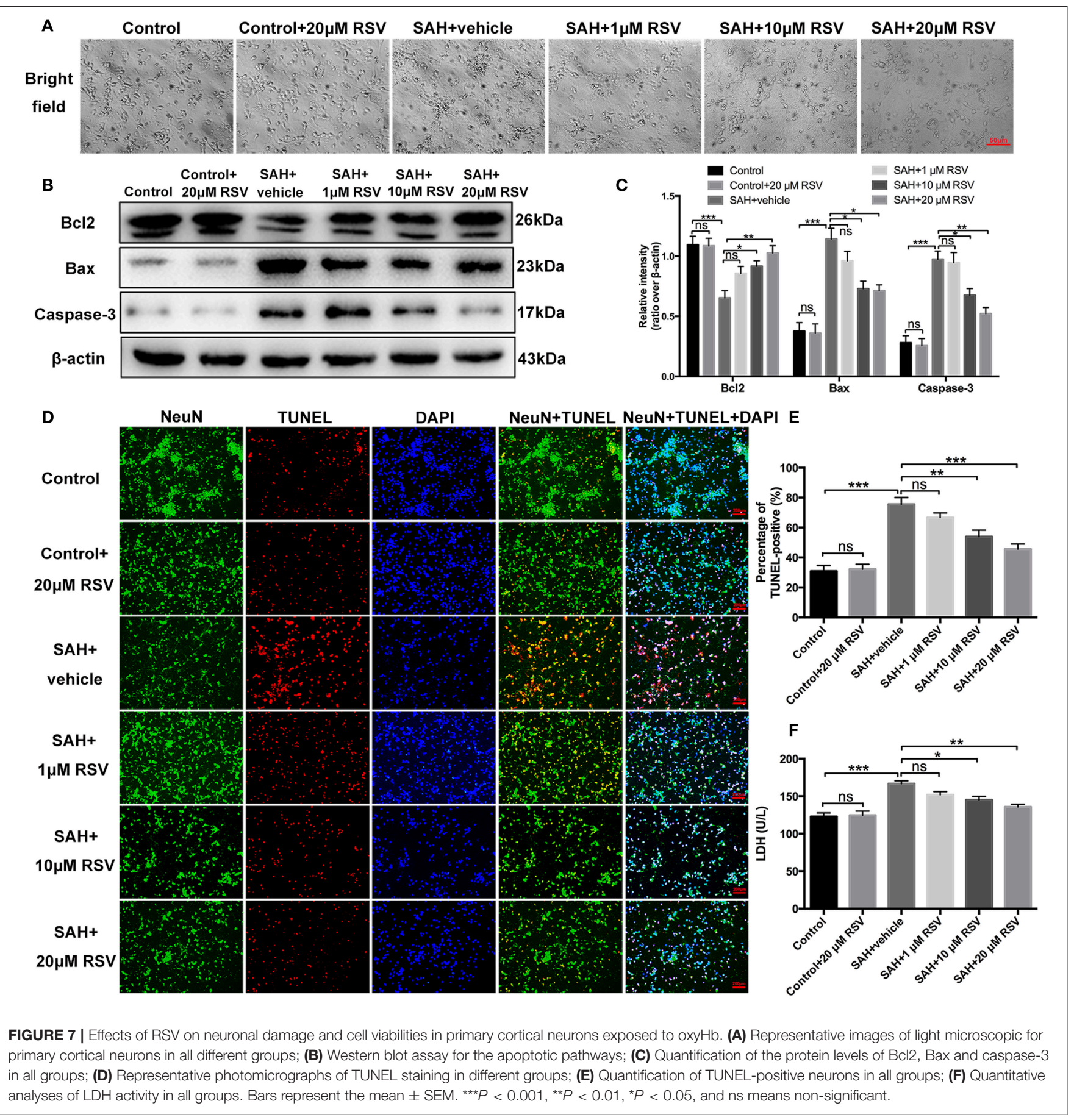

shed new light on the treatment of SAH, and suggest that RSV may be an attractive therapeutic agent in treatment of SAH.

\section{AUTHOR CONTRIBUTIONS}

XiaZ and QW wrote the paper. XiaZ, QW, YL, JL, and SL performed the experiments. XiaZ and WL contributed to the analysis of samples and data. QZ, MZ, CH, and XinZ designed the study and revised the manuscript. All authors approved the final version of the manuscript.

\section{ACKNOWLEDGMENTS}

This work was supported by grants from The National Natural Science Foundation of China (NSFC): No. 81371294 and 81771291 (CH), 81471183 (XinZ), 81401029 (WL), 81571162 (MZ), 81501022 (XiaZ), and 81771291 (CH), and the National Science Foundation of Jiangsu province: No. BK20141375 (CH) and BK20141377 (XinZ) and a research project funded by Jinling Hospital: No. 2015013 (XiaZ). 


\section{REFERENCES}

Arteaga, O., Revuelta, M., Uriguen, L., Alvarez, A., Montalvo, H., and Hilario, E. (2015). Pretreatment with resveratrol prevents neuronal injury and cognitive deficits induced by perinatal hypoxia-ischemia in rats. PLOS ONE 10:e0142424. doi: 10.1371/journal.pone.0142424

Chen, S., Feng, H., Sherchan, P., Klebe, D., Zhao, G., Sun, X., et al. (2014). Controversies and evolving new mechanisms in subarachnoid hemorrhage. Prog. Neurobiol. 115, 64-91. doi: 10.1016/j.pneurobio.2013.09.002

Chen, S., Ma, Q., Krafft, P. R., Hu, Q., Rolland, W. II, Sherchan, P., et al. (2013). $\mathrm{P} 2 \mathrm{X} 7 \mathrm{R} /$ cryopyrin inflammasome axis inhibition reduces neuroinflammation after SAH. Neurobiol. Dis. 58, 296-307. doi: 10.1016/j.nbd.2013.06.011

Coll, R. C., Robertson, A. A., Chae, J. J., Higgins, S. C., Munoz-Planillo, R., Inserra, M. C., et al. (2015). A small-molecule inhibitor of the NLRP3 inflammasome for the treatment of inflammatory diseases. Nat. Med. 21, 248-255. doi: 10.1038/nm.3806

Della-Morte, D., Dave, K. R., DeFazio, R. A., Bao, Y. C., Raval, A. P., and PerezPinzon, M. A. (2009). Resveratrol pretreatment protects rat brain from cerebral ischemic damage via a sirtuin 1-uncoupling protein 2 pathway. Neuroscience 159, 993-1002. doi: 10.1016/j.neuroscience.2009.01.017

Dong, Y., Fan, C., Hu, W., Jiang, S., Ma, Z., Yan, X., et al. (2016). Melatonin attenuated early brain injury induced by subarachnoid hemorrhage via regulating NLRP3 inflammasome and apoptosis signaling. J. Pineal Res. 60, 253-262. doi: 10.1111/jpi.12300

Fu, Y., Wang, Y., Du, L., Xu, C., Cao, J., Fan, T., et al. (2013). Resveratrol inhibits ionising irradiation-induced inflammation in MSCs by activating SIRT1 and limiting NLRP-3 inflammasome activation. Int. J. Mol. Sci. 14, 14105-14118. doi: 10.3390/ijms 140714105

Guo, Z. N., Xu, L., Hu, Q., Matei, N., Yang, P., Tong, L. S., et al. (2016). Hyperbaric oxygen preconditioning attenuates hemorrhagic transformation through reactive oxygen species/thioredoxin-interacting protein/nod-like receptor protein 3 Pathway in hyperglycemic middle cerebral artery occlusion rats. Crit. Care Med. 44, e403-e411. doi: 10.1097/CCM.0000000000001468

Koronowski, K. B., Dave, K. R., Saul, I., Camarena, V., Thompson, J. W., Neumann, J. T., et al. (2015). Resveratrol preconditioning induces a novel extended window of ischemic tolerance in the mouse brain. Stroke 46, 2293-2298. doi: 10.1161/STROKEAHA.115.009876

Lan, X., Han, X., Li, Q., Li, Q., Gao, Y., Cheng, T., et al. (2017a). Pinocembrin protects hemorrhagic brain primarily by inhibiting toll-like receptor 4 and reducing M1 phenotype microglia. Brain Behav. Immun. 61, 326-339. doi: 10.1016/j.bbi.2016.12.012

Lan, X., Han, X., Li, Q., Yang, Q. W., and Wang, J. (2017b). Modulators of microglial activation and polarization after intracerebral haemorrhage. Nat. Rev. Neurol. 13, 420-433. doi: 10.1038/nrneurol.2017.69

Lebeaupin, C., Proics, E., de Bieville, C. H., Rousseau, D., Bonnafous, S., Patouraux, S., et al. (2015). ER stress induces NLRP3 inflammasome activation and hepatocyte death. Cell Death Dis. 6:e1879. doi: 10.1038/cddis.2015.248

Li, J., Chen, J., Mo, H., Chen, J., Qian, C., Yan, F., et al. (2015). Minocycline protects against NLRP3 inflammasome-induced inflammation and P53-associated apoptosis in early brain injury after subarachnoid hemorrhage. Mol. Neurobiol. 53, 2668-2678. doi: 10.1007/s12035-015-9318-8

Li, Y., Yang, J., Chen, M. H., Wang, Q., Qin, M. J., Zhang, T., et al. (2015). Ilexgenin A inhibits endoplasmic reticulum stress and ameliorates endothelial dysfunction via suppression of TXNIP/NLRP3 inflammasome activation in an AMPK dependent manner. Pharmacol. Res. 99, 101-115. doi: $10.1016 /$ j.phrs.2015.05.012

Lin, C. J., Chen, T. H., Yang, L. Y., and Shih, C. M. (2014). Resveratrol protects astrocytes against traumatic brain injury through inhibiting apoptotic and autophagic cell death. Cell Death Dis. 5:e1147. doi: 10.1038/cddis.2014.123

Lopez, M. S., Dempsey, R. J., and Vemuganti, R. (2015). Resveratrol neuroprotection in stroke and traumatic CNS injury. Neurochem. Int. 89, 75-82. doi: 10.1016/j.neuint.2015.08.009

Ma, Q., Chen, S., Hu, Q., Feng, H., Zhang, J. H., and Tang, J. (2014). NLRP3 inflammasome contributes to inflammation after intracerebral hemorrhage. Ann. Neurol. 75, 209-219. doi: 10.1002/ana.24070

Murphy, M. P. (2009). How mitochondria produce reactive oxygen species. Biochem. J. 417, 1-13. doi: 10.1042/BJ20081386
Narayanan, S. V., Dave, K. R., Saul, I., and Perez-Pinzon, M. A. (2015). Resveratrol preconditioning protects against cerebral ischemic injury via nuclear erythroid 2-related factor 2. Stroke 46, 1626-1632. doi: 10.1161/STROKEAHA.115.008921

Rege, S. D., Geetha, T., Griffin, G. D., Broderick, T. L., and Babu, J. R. (2014). Neuroprotective effects of resveratrol in Alzheimer disease pathology. Front. Aging Neurosci. 6:218. doi: 10.3389/fnagi.2014.00218

Sehba, F. A., Hou, J., Pluta, R. M., and Zhang, J. H. (2012). The importance of early brain injury after subarachnoid hemorrhage. Prog. Neurobiol. 97, 14-37. doi: 10.1016/j.pneurobio.2012.02.003

Sehba, F. A., Pluta, R. M., and Zhang, J. H. (2011). Metamorphosis of subarachnoid hemorrhage research: from delayed vasospasm to early brain injury. Mol. Neurobiol. 43, 27-40. doi: 10.1007/s12035-010-8155-z

Shao, A., Wu, H., Hong, Y., Tu, S., Sun, X., Wu, Q., et al. (2015). Hydrogenrich saline attenuated subarachnoid hemorrhage-induced early brain injury in rats by suppressing inflammatory response: possible involvement of NF-symbolizeB pathway and NLRP3 Inflammasome. Mol. Neurobiol. 53, 3462-3476. doi: 10.1007/s12035-015-9242-y

Shao, A. W., Wu, H. J., Chen, S., Ammar, A. B., Zhang, J. M., and Hong, Y. (2014). Resveratrol attenuates early brain injury after subarachnoid hemorrhage through inhibition of NF-kappaB-dependent inflammatory/MMP-9 pathway. CNS Neurosci. Ther. 20, 182-185. doi: 10.1111/cns.12194

Sui, D. M., Xie, Q., Yi, W. J., Gupta, S., Yu, X. Y., Li, J. B., et al. (2016). Resveratrol Protects against Sepsis-Associated Encephalopathy and Inhibits the NLRP3/IL-1beta Axis in Microglia. Mediat. Inflamm. 2016:1045657. doi: $10.1155 / 2016 / 1045657$

Sun, Q., Wu, W., Hu, Y. C., Li, H., Zhang, D., Li, S., et al. (2014). Early release of high-mobility group box 1 (HMGB1) from neurons in experimental subarachnoid hemorrhage in vivo and in vitro. J. Neuroinflammation 11:106. doi: 10.1186/1742-2094-11-106

Wali, J. A., Masters, S. L., and Thomas, H. E. (2013). Linking metabolic abnormalities to apoptotic pathways in Beta cells in type 2 diabetes. Cells 2, 266-283. doi: 10.3390/cells2020266

Walsh, J. G., Muruve, D. A., and Power, C. (2014). Inflammasomes in the CNS. Nat. Rev. Neurosci. 15, 84-97. doi: 10.1038/nrn3638

Wang, F., Cui, N., Yang, L., Shi, L., Li, Q., Zhang, G., et al. (2015). Resveratrol Rescues the impairments of hippocampal neurons stimulated by microglial over-activation in vitro. Cell Mol. Neurobiol. 35, 1003-1015. doi: 10.1007/s10571-015-0195-5

Wang, Y., Kong, H., Zeng, X., Liu, W., Wang, Z., Yan, X., et al. (2016). Activation of NLRP3 inflammasome enhances the proliferation and migration of A549 lung cancer cells. Oncol. Rep. 35, 2053-2064. doi: 10.3892/or.2016.4569

Wang, Z., Shi, X. Y., Yin, J., Zuo, G., Zhang, J., and Chen, G. (2012). Role of autophagy in early brain injury after experimental subarachnoid hemorrhage. J. Mol. Neurosci. 46, 192-202. doi: 10.1007/s12031-011-9575-6

Wang, Z., Wang, Y., Tian, X., Shen, H., Dou, Y., Li, H., et al. (2016). Transient receptor potential channel $1 / 4$ reduces subarachnoid hemorrhage-induced early brain injury in rats via calcineurin-mediated NMDAR and NFAT dephosphorylation. Sci. Rep. 6:33577. doi: 10.1038/srep33577

Wu, J., Xu, X., Li, Y., Kou, J., Huang, F., Liu, B., et al. (2014). Quercetin, luteolin and epigallocatechin gallate alleviate TXNIP and NLRP3-mediated inflammation and apoptosis with regulation of AMPK in endothelial cells. Eur. J. Pharmacol. 745, 59-68. doi: 10.1016/j.ejphar.2014.09.046

Wu, L. Y., Ye, Z. N., Zhou, C. H., Wang, C. X., Xie, G. B., Zhang, X. S., et al. (2017). Roles of pannexin-1 channels in inflammatory response through the TLRs/NFkappa B signaling pathway following experimental subarachnoid hemorrhage in rats. Front. Mol. Neurosci. 10:175. doi: 10.3389/fnmol.2017.00175

Wu, Q., Qi, L., Li, H., Mao, L., Yang, M., Xie, R., et al. (2017). Roflumilast reduces cerebral inflammation in a rat model of experimental subarachnoid hemorrhage. Inflammation. 40, 1245-1253. doi: 10.1007/s10753-0170567-8

Yan, H., Zhang, D., Hao, S., Li, K., and Hang, C. H. (2015). Role of mitochondrial calcium uniporter in early brain injury after experimental subarachnoid hemorrhage. Mol. Neurobiol. 52, 1637-1647. doi: 10.1007/s12035-014-8942-z

Yang, F., Wang, Z., Wei, X., Han, H., Meng, X., Zhang, Y., et al. (2014). NLRP3 deficiency ameliorates neurovascular damage in experimental ischemic stroke. J. Cereb. Blood Flow Metab. 34, 660-667. doi: 10.1038/jcbfm.2013.242 
Yang, S. J., and Lim, Y. (2014). Resveratrol ameliorates hepatic metaflammation and inhibits NLRP3 inflammasome activation. Metabolism 63, 693-701. doi: 10.1016/j.metabol.2014.02.003

Yang, X., Xu, S., Qian, Y., and Xiao, Q. (2017). Resveratrol regulates microglia M1/M2 polarization via PGC-1alpha in conditions of neuroinflammatory injury. Brain Behav. Immun. 64, 162-172. doi: 10.1016/j.bbi.2017.03.003

You, W., Wang, Z., Li, H., Shen, H., Xu, X., Jia, G., et al. (2016). Inhibition of mammalian target of rapamycin attenuates early brain injury through modulating microglial polarization after experimental subarachnoid hemorrhage in rats. J. Neurol. Sci. 367, 224-231. doi: 10.1016/j.jns.2016.06.021

Yuan, J., Liu, W., Zhu, H., Zhang, X., Feng, Y., Chen, Y., et al. (2017). Curcumin attenuates blood-brain barrier disruption after subarachnoid hemorrhage in mice. J. Surg. Res. 207, 85-91. doi: 10.1016/j.jss.2016.08.090

Zhang, Q., Yuan, L., Zhang, Q., Gao, Y., Liu, G., Xiu, M., et al. (2015). Resveratrol attenuates hypoxia-induced neurotoxicity through inhibiting microglial activation. Int. Immunopharmacol. 28, 578-587. doi: 10.1016/j.intimp.2015.07.027

Zhang, T., Su, J., Wang, K., Zhu, T., and Li, X. (2014). Ursolic acid reduces oxidative stress to alleviate early brain injury following experimental subarachnoid hemorrhage. Neurosci. Lett. 579, 12-17. doi: 10.1016/j.neulet.2014.07.005

Zhang, X. S., Li, W., Wu, Q., Wu, L. Y., Ye, Z. N., Liu, J. P., et al. (2016a). Resveratrol attenuates acute inflammatory injury in experimental subarachnoid hemorrhage in rats via inhibition of TLR4 pathway. Int. J. Mol. Sci. 17:e1131. doi: 10.3390/ijms17081331

Zhang, X. S., Wu, Q., Wu, L. Y., Ye, Z. N., Jiang, T. W., Li, W., et al. (2016b). Sirtuin 1 activation protects against early brain injury after experimental subarachnoid hemorrhage in rats. Cell Death Dis. 7:e2416. doi: 10.1038/cddis.2016.292

Zhang, X. S., Zhang, X., Zhang, Q. R., Wu, Q., Li, W., Jiang, T. W., et al. (2015). Astaxanthin reduces matrix metalloproteinase- 9 expression and activity inthe brain after experimental subarachnoid hemorrhage in rats. Brain Res. 1624, 113-124. doi: 10.1016/j.brainres.2015.07.020

Zhang, X. S., Zhang, X., Zhou, M. L., Zhou, X. M., Li, N., Li, W., et al. (2014). Amelioration of oxidative stress and protection against early brain injury by astaxanthin after experimental subarachnoid hemorrhage. J. Neurosurg. 121, 42-54. doi: 10.3171/2014.2.JNS13730

Zhang, Z., Zhang, Z., Lu, H., Yang, Q., Wu, H., and Wang, J. (2017). Microglial polarization and inflammatory mediators after intracerebral hemorrhage. Mol. Neurobiol. 54, 1874-1886. doi: 10.1007/s12035-0169785-6

Zhou, X., Chen, M., Zeng, X., Yang, J., Deng, H., Yi, L., et al. (2014). Resveratrol regulates mitochondrial reactive oxygen species homeostasis through Sirt3 signaling pathway in human vascular endothelial cells. Cell Death Dis. 5:e1576. doi: $10.1038 /$ cddis. 2014.530

Zhu, W., Cao, F. S., Feng, J., Chen, H. W., Wan, J. R., Lu, Q., et al. (2017). NLRP3 inflammasome activation contributes to long-term behavioral alterations in mice injected with lipopolysaccharide. Neuroscience 343, 77-84. doi: 10.1016/j.neuroscience.2016.11.037

Conflict of Interest Statement: The authors declare that the research was conducted in the absence of any commercial or financial relationships that could be construed as a potential conflict of interest.

Copyright (c) 2017 Zhang, Wu, Zhang, Lu, Liu, Li, Lv, Zhou, Zhang and Hang. This is an open-access article distributed under the terms of the Creative Commons Attribution License (CC BY). The use, distribution or reproduction in other forums is permitted, provided the original author(s) or licensor are credited and that the original publication in this journal is cited, in accordance with accepted academic practice. No use, distribution or reproduction is permitted which does not comply with these terms. 